\title{
The Impact of Different Game Types and Sports on College Students' Physical Activity and Motivation in Basic Instruction Program Settings
}

\author{
Yang Song ${ }^{1 *}$, Stephen Harvey ${ }^{2}$, James Hannon ${ }^{3}$, Karen Rambo-Hernandez ${ }^{4}$, Emily Jones ${ }^{5}$, Sean Bulger ${ }^{6}$ \\ ${ }^{I}$ The Division of Liberal Studies and Education, Lane College, Jackson, Tennessee, United States \\ ${ }^{2}$ Patton College of Education, Department of Recreation and Sport Pedagogy, Ohio University Athens, Ohio, United States \\ ${ }^{3}$ College of Education, Health and Human Services, Kent State University Kent, Ohio, United States \\ ${ }^{4}$ College of Education and Human Services, West Virginia University Morgantown, West Virginia, United States \\ ${ }^{5}$ College of Applied Science and Technology, Illinois State University Normal, Illinois, United States \\ ${ }^{6}$ College of Physical Activity and Sport Sciences, West Virginia University Morgantown, West Virginia, United States
}

Corresponding Author: Yang Song, E-mail: ysong@lanecollege.edu

\begin{tabular}{l} 
ARTICLE INFO \\
\hline Article history \\
Received: September 12, 2018 \\
Accepted: October 25, 2018 \\
Published: October 31, 2018 \\
Volume: 6 Issue: 4
\end{tabular}

Conflicts of interest: None Funding: None

\begin{abstract}
Background: Although Basic Instruction Program (BIP) or Higher Education Physical Activity Program (HEPAP) classes within university/colleges are founded on the rationale of providing students with opportunities to be physically active, little is known about the physical activity (PA) accrual and/or motivation levels in these classes. Objective: The purpose of the present study was to investigate college students' Moderate to Vigorous Physical Activity (MVPA) and motivation levels while playing different games types (modified games/MGs, small-sided games/SSGs, and full-sided games/FSGs) in badminton and soccer classes. In addition, the study examined the extent to which motivation levels predicted students MVPA. Method: Participants were seventyone college students (14 females) from a rural Mid-Atlantic university in the United States (U.S.). Triaxial accelerometers were used to collect MVPA data and the Intrinsic Motivation Inventory (IMI) to measure student motivation. Results: Students in soccer classes had statistically significant more MVPA than those in badminton. Students in soccer and badminton classes had most MVPA in FSGs and SSGs, respectively. Although students reported similar scores in the three IMI subscales when data were aggregated, soccer students reported higher levels of competence and effort in MGs, whereas badminton students reported higher levels of competence and effort in SSGs. Interest was the only statistically significant predictor of MVPA in MGs whereas perceived competence statistically significantly predicted MVPA in both SSGs and FSGs. Conclusion: PE and sport practitioners should utilize the different game types to find the optimal balance between MVPA and student motivation and realize lesson objectives.
\end{abstract}

Key words: Universities, Students, Exercise, Motivation, Soccer, Racquet sports, Accelerometry

\section{INTRODUCTION}

Healthy People (2020) provides the physical activity guidelines for adults in aerobic physical activity (at least $150 \mathrm{~min}$ utes/week at moderate intensity or 75 minutes/week at vigorous intensity) and in muscle-strengthening activity (two or more days a week). However, only about $20 \%$ of adults meet the guidelines for both aerobic and muscle-strengthening activity (Healthy People, 2020). With about 20 million students served in U.S. colleges and universities (American College Health Association, 2012), college/university students, a major source of future generations of parents and policy-makers, should be a research targeted population for enhancing the proportion of adults achieving the Healthy People 2020 physical activity goals. Fortunately, most col- leges/universities provide their students with physical activity (PA) opportunities in the form of a Basic Instruction Program (BIP) or Higher Education Physical Activity Program (HEPAP) (Stapleton, Taliaferro, \& Bulger, 2017). It is crucial for adult learners to develop the appreciation for physical activity in BIPs or HEPAP because physical activity behaviors established during the college years will persist into adulthood (Sparling \& Snow, 2002). To encourage student participation in BIP or HEPAP classes, many colleges/universities offer a variety of classes in activities and sports that students will have previously participated in, either recreationally or within a competitive context, during high school. However, despite BIP and HEPAP classes being founded on the rationale of providing college students with opportunities to be physically active during their time at university, little is 
known about how these classes assist students in meeting slated PA goals. In addition, although the Institute of Medicine (IOM, 2013) has recommended k-12 students engage in moderate to vigorous physical activity (MVPA) levels for at least 50 percent of PE class time, no such stipulations have been afforded to university BIP/HEPAP classes. Moreover, it is clear that the nature of the activity being conducted within these BIP/HEPAP classes could affect the amount of PA accumulation during one session. Emerging research in sport and physical education research, for example, suggests that using a variety of smaller-sided games (SSG's), and/ or modified games (MGs) as opposed to full-sided games (FSGs) may affect participant PA levels. For the purpose of the study, the definitions of the following terms were taken from Roberts \& Fairclough (2012). Modified games (MGs): the class is engaged in a modified game. Modification of the game includes rules (e.g., the ball or the projectile is not allowed over a certain distance, height), conditions and equipment (e.g., throw-catch badminton, alternative scoring zones). The game reduces the dominance of skills and techniques. The numbers in the team must be equal for it to be considered a game (1 vs. 1, 2 vs. 2, 3 vs. 3, 4 vs. 4 ) and not an overload practice. Small-sided games (SSGs): the class is engaged in SSGs with no conditions. For example, a 1 vs. 1 half-court game of badminton, which uses regulation size rackets/shuttlecocks and there is no restriction on the skills and techniques, i.e. smashing; or, a 6 vs. 6 small-sided soccer game with no conditions other than the numbers on the playing area and adaptations to the pitch/court size. Full-sided games (FSGs): the class is involved in a full version of the game including numbers and pitch/court size.

In contrast to the benefits of MGs and SSGs, physical education (PE) researchers have demonstrated that frequent usage of FSGs would result in low MVPA. For example, Roberts and Fairclough (2011) found that students were mostly inactive during class time. They believed that the overuse of FSGs ( $21 \%$ of class time), compared to $4 \%$ class time of MGs, led to the low PA levels. Sport researchers have previously investigated the effects of the number of players and pitch sizes on students'/players' PA levels (e.g., Bell, Johnson, Shimon, \& Bale, 2013; Rampinini et al., 2007). In general, these studies have demonstrated that game formats with fewer players elicit more PA levels than the game format with more players. For example, Rampinini et al. (2007) found that soccer players in a 3-a-side game could achieve $87-90 \%$ heart rate (HR) max range, whereas, players in a 5 -a-side game could only achieve $82-87 \%$ HR max range. Based on the findings of those studies, students are more active in MGs and SSGs than they are in FSGs. Despite these trends in PE and sport research, little to no previous research in BIP/HEPAP contexts has examined PA accumulations, exclusively in game play period.

Previous research (e.g., Carroll \& Loumidis, 2001) has demonstrated the strong correlations between students' propensity to engage in PA in PE and their motivation. Understanding students' motivation for PE and sport is helpful for PE and sport practitioners so they can adjust session to meet participant needs, thus, enhance their motivation, and therefore PA levels. Self-determination theory (SDT; Deci \&
Ryan, 1985) is a general psychology theory to explain human behaviors and it provides a valuable framework to understand students' motivation in PE. SDT assumes that when the three basic needs are met: autonomy (having choices), competence (abilities to control outcomes), and relatedness (involvement and connection with others), students are more likely to demonstrate higher levels of self-determined motivation, which would lead to positive intentions to participate in PE (e.g., Sun \& Chen, 2010).

SDT posits that behaviors are driven by both intrinsic motivations and extrinsic motivations. The core of SDT is the provisions to satisfy the three psychological needs and the progressions from amotivation, extrinsic motivation, to intrinsic motivation. Intrinsic motivation refers to the engagement in a behavior for the sake of the behavior itself. Extrinsic motivation refers to the engagement in a behavior for the sake of other benefits. For example, when a boy chooses to play soccer, he is intrinsically motivated if he does so for the love of playing soccer, but he is extrinsically motivated if he treats playing soccer only as a way to get involved with his friends. The last form of motivation is amotivation, which means that a person is neither intrinsically nor extrinsically motivated. Based on SDT, the quality of motivation is on a continuum ranging from amotivation all the way up to intrinsic motivation.

Many instruments have been designed to measure participants perceived levels of self-determined motivation related to a target activity in experimental psychology research. The Intrinsic Motivation Inventory (IMI; Ryan, 1982) is one such instrument. The original IMI includes six subscales. They are: (1) interest/enjoyment, (2) perceived competence, (3) effort, (4) value/usefulness, (5) pressure and tension, and (6) perceived choice. A seventh subscale, relatedness, was subsequently added. The IMI subscale items have been shown to be valid through factorial analysis studies (Deci \& Ryan, 2003). The authors of the current study only focused on the first three subscales. Some of the subscales were not selected since they did not fit the current study purposes. For example, students were assigned to play three games that were pre-determined by the researchers, thus, the perceived choice subscale does not fit. The authors also did not put students in a situation where they would experience significant pressures during any of the games.

Even though researchers have conducted numerous studies concerning students' PA or MVPA in Game-Centered Approach interventions (Harvey et al., 2016), to our knowledge, no studies have examined university students' PA levels and motivations when they participate in different game types (SSGs, MGs, and FSGs) across two different sports - badminton and soccer. Badminton and soccer are one of the most popular net/wall games and invasion games in the world, respectively (Almond, 1986). Although Ainsworth et al. (2011) previously found that adults would get more MVPA from soccer than badminton it is still necessary to investigate MVPA differences in the current study. First, Ainsworth and colleagues utilized subjective self-reported questionnaires to collect PA data, while in this current study accelerometers will be used. Heyward and Gibson (2014) stated that accelerometers are the best devices to measure 
PA levels objectively because of their ability to monitor PA minute-by-minute, differentiate intensity levels, are feasible with people of all ages, are accurate with static and dynamic behaviors, and hold large amounts of data memory. Second, Ainsworth and colleagues only collected PA data only in regular games abiding by the official rules and court sizes rather than across different game types (SSGs, MGs, and GSGs) which may affect MVPA differently. The purpose of this study was to investigate college-aged students' PA levels and motivation in different game types (i.e., MGs, SSGs, and FSGs) and in different sports (badminton and soccer). The current study will answer the following three research questions: a) how do students' MVPA levels differ in the different game types and sports; b) how do students' motivation levels differ in different game types and sports; and, c) to what extent do motivation levels predict PA levels. The findings of this study will provide PE and sport practitioners with knowledge about the likely MVPA levels derived from MGs, SSGs, and FSGs, and with knowledge about students' motivations toward the different game types so they can be judiciously applied in their respective instructional settings.

\section{METHOD}

\section{Participants and Setting}

One hundred and twelve students from a rural mid-Atlantic University in the U.S. initially participated the study. Seventy-one students between the ages of 18 and 39 years old $(M=19.6, S D=3.1)$ completed MVPA data collection, and 67 of them finished the MVPA and IMI data collection. The students were from three soccer classes (40 males and 8 females) and two badminton classes (17 males and 6 females). This sample size was verified based on power calculations for a significant effect $(p=.05)$ at an effect size of 0.35 , with a repeated measures, within-between interaction, F test MANOVA conducted for 5 groups (five classes), with three repetitions (types of games were played) using the G-Power 3.0.10 software (Erdfelder, Faul, \& Buchner, 1996). The activity classes were general electives and open to the whole university community. All the classes lasted five weeks and classes met either three times a week (75 minutes per class) or twice a week (100 minutes per class). Before recruiting participants, the University Institutional Review Board (IRB) protocol was submitted and approved. All participants signed consent forms and received a data collection schedule. Students did not receive extra credit or penalties for choosing to participate or not participate in the study. A Graduate Teaching Assistant (GTA) taught all three soccer classes. A different GTA taught the two badminton classes.

\section{Research Design}

The study was a cross-sectional quasi-experimental design. It was a cross-sectional design since both MVPA data and IMI data were collected from three soccer classes and two badminton classes in three-game type conditions: modified-games (MGs), small-sided games (SSGs), and full-sided games (FSGs). It was a quasi-experimental design because students chose the classes on their own, and, therefore, were not randomly assigned to the two sports. Participants played the three game types in a fixed order. They played the MGs first, SSGs second, and FSGs third. Participants played each of the games for 15 minutes on three different days of class.

\section{Procedure}

No badminton classes were available for a pilot study; thus, a pilot study was conducted only in soccer MGs and SSGs. However, the purpose of familiarizing the researchers with the data collection instruments was achieved. When it was the time for the formal study, the lead researcher held meetings with the two GTAs to inform them about the data collection schedules and the game types. Moreover, the researcher stressed the importance to the two GTAs that researchers would not intervene in how they teach the classes except for embedding the three games on data collection days. GTAs of the university used the Sport Education model to teach activity classes (Meeteer, Housner, Bulger, Hawkins, \& Wiegand, 2012). Thus, using games in activity classes was a part of their already existing teaching repertoire. The request of having students play the three games on different days was a natural fit for the class schedule. Moreover, GTAs were required not to provide any instructions, feedback, or encouragement when students were playing the games. Researchers found that interactions between coaches with players would positively enhance players' MVPA (e.g., Coutts et al., 2004; Hoff et al., 2002;). The non-interaction requirement would eliminate this influence.

Given the fact that GTAs spend most of the first class in administration duties, the researchers attended the second class to request the students' participation in the current study. After students received and signed consent forms, researchers collected necessary anthropometric data to assign accelerometers to them. The required data for programming accelerometers was: gender, date of birth, weight (measured a bioimpedance scale OMRON HBF-516B), height (measured by a portable stadiometer CHARDERHM-200P Portstad), dominant side, and race.

On the specific data collection days, participants put their accelerometers on at the beginning of the class. After the warm-up, GTAs introduced the game types to students by explaining the games and having them play the game type (i.e., MGs, SSGs, and FSGs) for five minutes for familiarity purposes. Immediately after playing, GTAs provided opportunities for participants to ask questions about the games. GTAs taught content in accordance with their planning for the rest of the class before having students play the official non-stop 15-minute games. To keep students playing the whole time, the GTAs granted students a two-minute water break right before the official gameplay. The researcher utilized a digital stopwatch to record the time. The researcher reminded the participants of the remaining game time at 10-minute, 5-minute, 3-minute and 1-minute time points.

Participants in the badminton classes played the MGs and SSGs using half of the court with the middle line as one sideline and the doubles sideline as another sideline (white or grey part of Figure 1). The only difference between the badminton MGs and SSGs was that students were not allowed to utilize smashes 


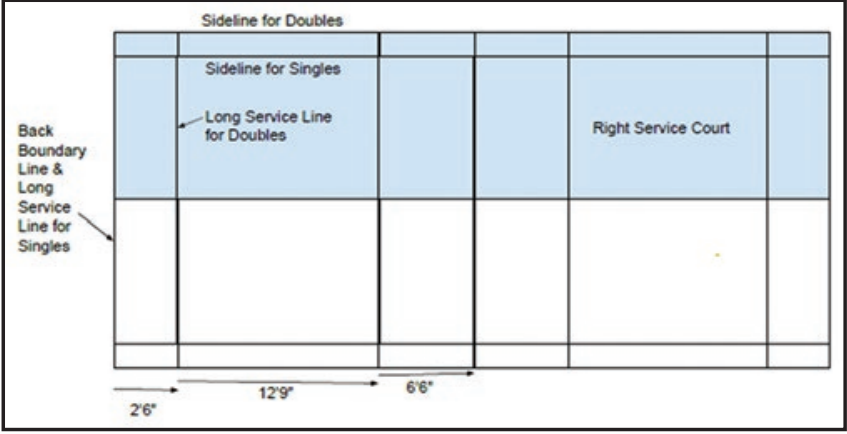

Figure 1. Badminton MSGs, SSGs, and FSGs

in MGs, whereas, they were permitted to utilize all the skills they could perform in SSGs. Four students played the MGs and SSGs at the same time. In other words, two games happened simultaneously on a court. To reduce the time to retrieve shuttlecocks, three shuttlecocks were stood on the outer sidelines, but far away from the court, so as not to cause injury. Participants played a regulation full-court singles game as FSGs.

Participants in the soccer classes played the 6 vs. 6 MGs and 7 vs. 7 SSGs on a pitch size of 50 yards x 40 yards (black or white half of Figure 2), which is the medium size of the recommend field size for US youth soccer 6 vs. 6 (US Youth Soccer, 2012). Another reason for choosing this size was the ability to have two MGs or SSGs arranged at the same time. In the soccer MGs, tall cones made up two small goals placed evenly on the sidelines. Each of the goals was six feet wide. In the SSGs, one regular size goal was placed in the center of each of the sideline. The main differences between MGs and SSGs in soccer were that no goalkeepers were present in MGs, but one goalkeeper defended a goal in SSGs. To keep 6 vs. 6 formats in both MGs and SSGs, even though it was 7 vs.7 in SSGs, researchers did not collect data from goalkeepers in the SSGs. Participants played the FSGs on a pitch size of 120 yards x 73 yards, which was the marked size on the field. Similar to placing extra shuttlecocks on the badminton sidelines, two soccer balls were placed evenly on each of the sidelines on the soccer field.

Immediately after playing each of the game types, participants were provided with the IMI paper questionnaires and pencils to fill out the questionnaires, which were originally created by the Qualtrics system. The first author brought paper versions of the questionnaire to each of the classes to avoid the situation where some students may not have been able to complete the questionnaire electronically. The first author collected the paper questionnaires, immediately transferring these paper versions of the IMI data to the Qualtrics system. After the initial data input, the researcher double-checked the accuracy of the transfer of paper records to the online system by exporting the each of the electronic datasets and double checking these against the initial paper records.

\section{Instrumentation}

\section{Physical activity data.}

Actigraph GT3X triaxial accelerometers (validated by Kelly et al., 2013) were used to collect students' PA data. Follow-

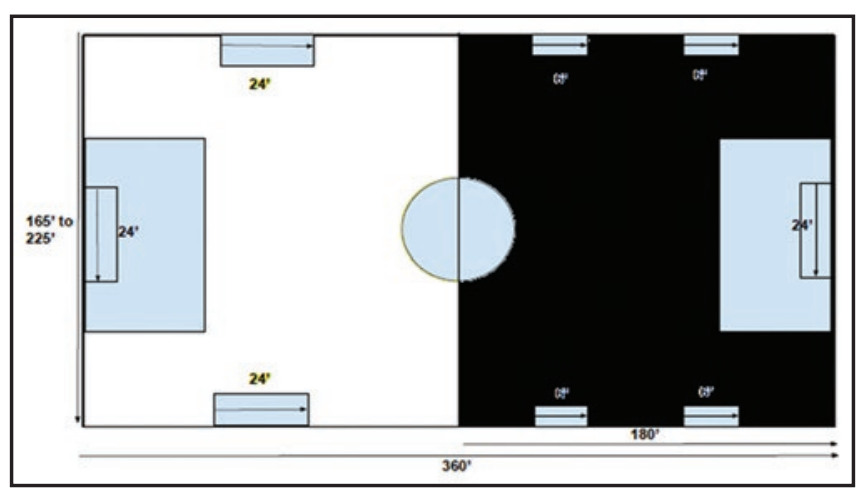

Figure 2. Soccer MSGs, SSGs, and FSGs

ing procedures outlined by Harvey et al., (2016), on data collection days, accelerometers were placed in a clear plastic bag that had the participants' ID number written on the outside, which corresponded with the ID number of the accelerometer inside the bag. Before the start of the session all participants attached the accelerometers around the waist of the dominant side of their body. Assistance was provided by researchers where required. This procedure was tested during a previous session before the study began. Once the data collection session was completed, each device was placed back into the correct bag and taken back to the lead researcher's office where the data on the devices were downloaded onto a laptop computer via the Actigraph software. The utilization of the Actigraph software permitted GT3X activity counts for each game at a 1-second epoch (Harvey et al., 2016). Data were extracted by applying a filter with the specific times of the lesson, which had previously been noted during data collection. This enabled the mean percentage of time spent in MVPA to be calculated using the Troiano et al. (2008) cut-off points for adults (2020 counts per minute) housed within the Actigraph software. These MVPA data were imported into Version 21 of SPSS (SPSS Inc, Chicago, IL) for statistical analyses.

\section{Intrinsic motivation inventory}

The IMI is a multidimensional measurement instrument designed for assessing participants' subjective experience related to a specific activity (e.g. Ryan, Mims, \& Koestner, 1983; Deci, Eghrari, Patrick, \& Leone, 1994). McAuley, Duncan, and Tammen (1989) validated the use of the IMI in PE settings. The original IMI includes six subscales, and include: (a) interest/enjoyment, (b) perceived competence, (c) effort, (d) value/usefulness, (e) pressure and tension, and (f) perceived choice. A seventh subscale, relatedness, was later added. Data were collected on the scales of interest/ enjoyment ( 7 items), perceived competence (6 items), and effort/importance ( 5 items). The IMI data were collected on a 7-point Likert scale ranging from $1=$ "not true at all" to 7 = "very true". There are two steps in scoring the IMI. First, items that were reverse scored were recoded. Second, subscale scores were calculated by averaging the scores across all the items on that subscale for each participant. The subscale scores were then used in the analyses of relevant questions. Reliability tests were conducted for each of the 
subscales, with results showing high reliability on each of the three sub-scales: interest/enjoyment ( $\alpha=.93)$, perceived/ competence $(\alpha=.94)$, effort/importance $(\alpha=.91)$.

\section{Data Analyses}

A two-way repeated measures ANOVA was utilized to test whether statistically significant MVPA differences existed among students from soccer classes and badminton classes (the main effect of sports on MVPA) and whether statistically significant MVPA differences existed among the three different game types (the main effect of game types on MVPA). The dependent variable was MVPA. The two independent variables were sports and game types. Interactions between the sports and game types are also reported if the interactions were statistically significant. Based on Green-Geisser Correction, F $(1.84,126.95)=3.02, \mathrm{p}=.06$, two separate one-way repeated measures ANOVA with Bonferroni correction ( $p=.03$ ) were also utilized to test whether MVPA differed in the three games forms for each sport (i.e., badminton and soccer). A two-way repeated measures MANOVA was utilized to test whether statistically significant differences existed in the three IMI sub-scales between the two sports and the three different game types. The dependent variables were the three IMI subscales (enjoyment, perceived competence, and effort), while the two independent variables were sports and game types. Statistically significant interactions are also reported.

Multiple regressions (where the grand mean centering technique was employed) were used to investigate the extent to which IMI scores and sports predicted MVPA, with one regression performed for each game form. The dependent variable was MVPA and the independent variables were IMI motivation (enjoyment/interest, perceived competence, and effort) and sports (badminton and soccer). All regression tests were conducted in two steps. The first step (Model 1) was completed by entering only the sports variable (badminton or soccer) and the second step (Model 2) involved entering both the sports variable and the three motivation subscale variables concurrently. With the addition of the motivation variables, the change of predicted variance of the dependent variables brought by the motivation data can be explained.

\section{RESULTS}

In this section, results pertaining to each of the three different research questions will be reported.

\section{Research question 1. How do students' MVPA levels differ in the different game types and sports?}

Based on findings from a split plot $\operatorname{ANOVA}(F(1,69)=29.1$; $p<.01)$, on average, students had statistically significantly more MVPA in soccer $(M=51.17 ; S D=1.81)$ than they did in badminton $(M=34.04 ; S D=2.61)$ (Figure 3). Moreover, when data from the two sports were combined, students attained similar MVPA in the three-game types (Figure 4). However, the interaction between game types and sports was

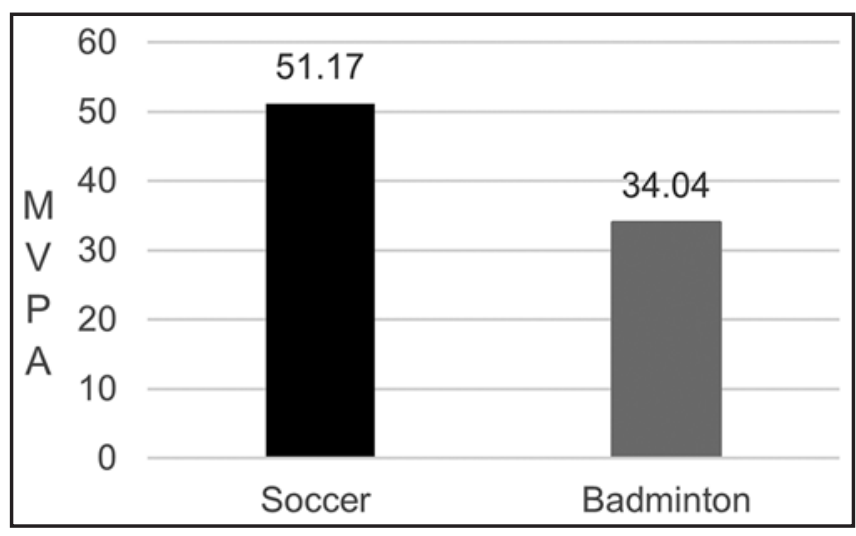

Figure 3. Aggregated MVPA data in soccer and badminton classes

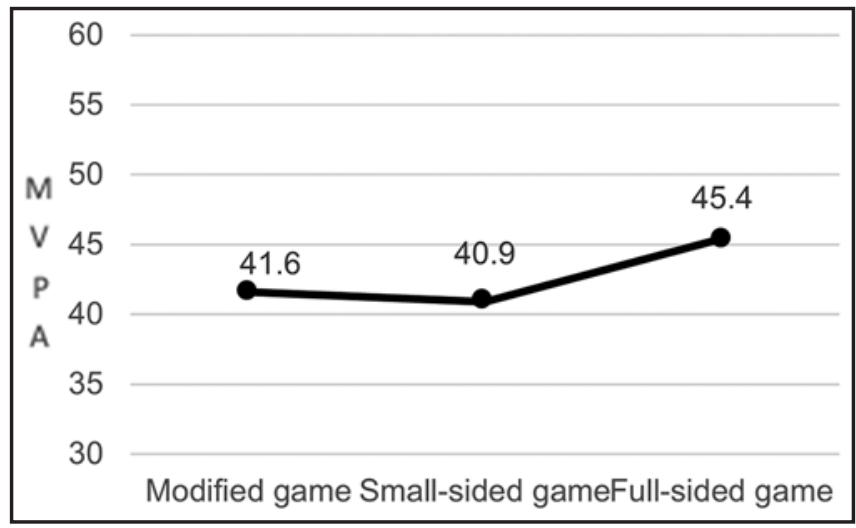

Figure 4. Aggregated MVPA data in different game types

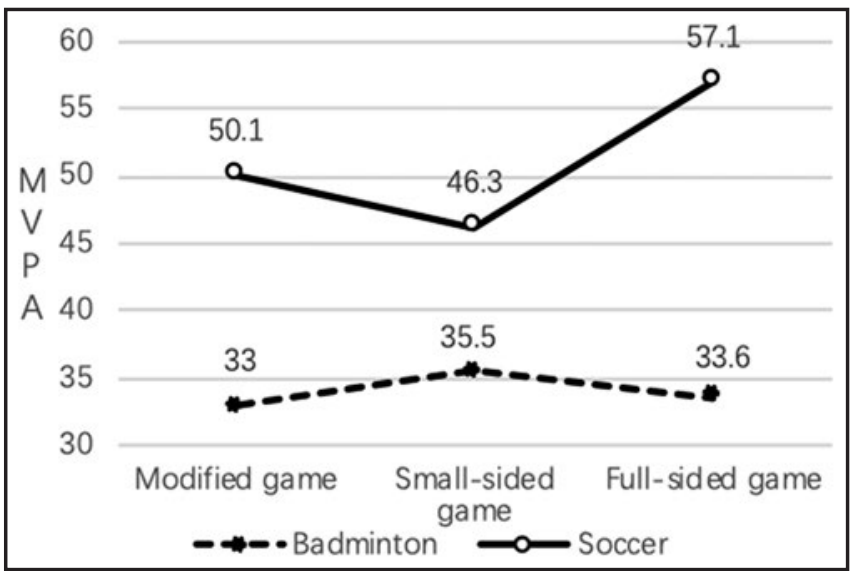

Figure 5. Badminton and soccer MVPA data in different game types

statistically significant $(p<.05)$. For example, students in soccer classes attained the most MVPA in FSGs and the least MVPA in SSGs. In contrast, students in badminton classes attained the most MVPA in SSGs and the least MVPA in MGs (Figure 5).

Based on findings from a one-way repeated measures ANOVA, students in the badminton classes had similar MVPA in the three games $(F(2,44)=.461, p>.05)$. However, students in the soccer classes had similar MVPA in MGs and SSGs, but statistically significantly more MVPA in FSGs $(F(1.715,80.594)=10.60, p<.001)$ when compared to MGs and SSGs. 
Research question 2. How do students' motivation levels differ in different game types and sports?

Findings from the two-way repeated measures MANOVA revealed there were no statistically significant differences in motivation between the two sports (Table 1). For example, students from both classes viewed the games as interesting, with students scoring more than 5.2 points in the interest/enjoyment scale. Moreover, there were no statistically significant differences in interest/enjoyment and perceived competence among the games. However, students perceived that they put statistically significantly more effort in SSGs and FSGs than that they did in MGs (Table 1).

Despite there being no statistically significant motivation differences between the two sports, the interaction patterns between the games and sports were statistically significant in the perceived competence subscale $(F(2,130)=4.3 ; p<.05)$. Students in badminton classes felt the most competent in SSGs games and the least competent in MGs. In contrast, students in soccer classes felt the most competent in MGs and the least competent in FSGs (Figure 6). In addition, the interaction between the games and sports was significant in terms of effort/importance $(F(2,130)=8.6 ; p<.01)$, with the interaction patterns the same as those seen in the perceived competence subscale. Students in badminton classes perceived they put the greatest effort into SSGs and least effort in MGs. Students in soccer classes perceived they put the greatest effort in MGs and least effort in FSGs (Figure 7).

\section{Research question 3. To what extent do motivation levels predict PA levels?}

Three multiple regressions were conducted to answer the research question with a regression for each of the game types (Table 2, Table 3, and Table 4). All the regressions were conducted with two steps. The first step was entering only the sports variable (badminton or soccer, Model 1) and the second step was entering both the sports variable and the three grand-mean centered motivation variables at the same time (Model 2).

The two-step entering helped detect the $R$ square change brought by the addition of the three motivation variables. Collinearity statistics Variance Inflation Factors (VIF) ranged from 1.03 to 2.46 , which was below the multicollinearity criterion (10), thus all the variables were maintained for subsequent analysis. In the regression models for MGs (Table 2), Sports was a statistically significant variable at $p=.01$ level, and it accounted for $20 \%$ of the variance of the dependent variable-MVPA. Based on the model, students in soccer classes had 17.63 units MVPA more than those in badminton classes (dummy coded as 0 ). When the three motivation variables were added into the model, the whole model accounted for $29 \%$ of the variance of the dependent variable. However, the addition of the motivation data did not make the change statistically significant. The interest/enjoyment variable was a statistically significant variable at a $p=.05$ level to predict MVPA. Based on the model, while holding all the other variables constant, every unit increase in interest/enjoyment would decrease 5.76 units MVPA. Sports was a statistically significant variable $(p<.01)$ in the regression models for SSGs (Table 3) and it accounted for $12 \%$ of the variance of dependent variable-MVPA. Based on the model, students in soccer classes had 12.17 units MVPA more than those in badminton classes. When the three motivation variables were added into the model, the whole model accounted for $36 \%$ of the variance of the dependent variable. Moreover, the addition of the motivation variables made the change statistically significant at the $p=.01$ level. In the whole model (model 2), both the sports and perceived competence variables were sta-

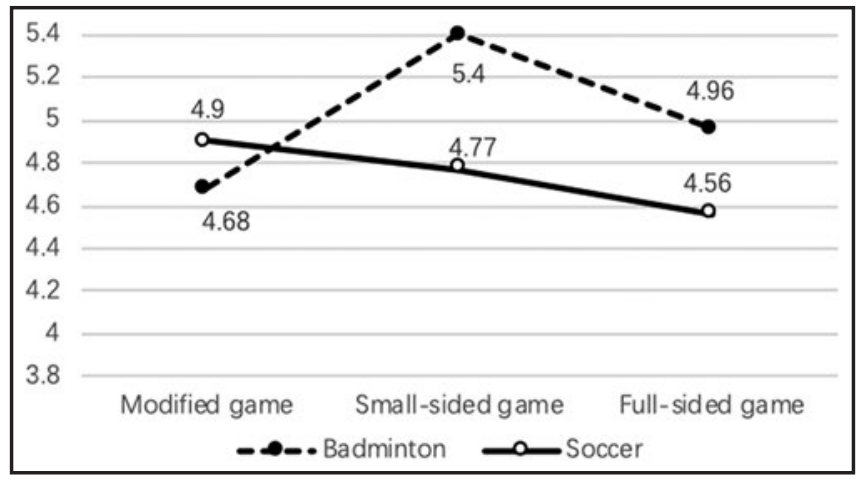

Figure 6. Perceived competence in game types and sports

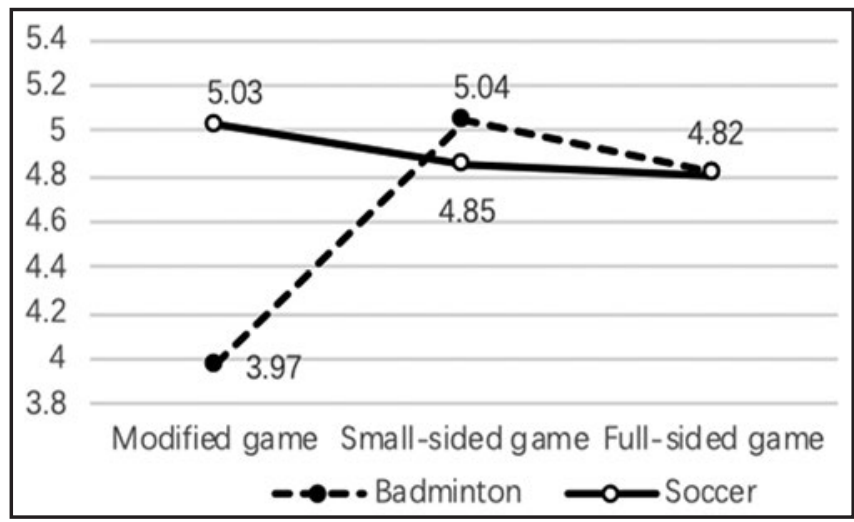

Figure 7. Effort/importance in game types and sports

Table 1. Motivation Data in Different Sports and Different Game Types

\begin{tabular}{|c|c|c|c|c|c|c|c|c|c|c|}
\hline \multirow[t]{3}{*}{ Motivation } & \multicolumn{4}{|c|}{ Sports } & \multicolumn{6}{|c|}{ Game Types } \\
\hline & \multicolumn{2}{|c|}{ Badminton } & \multicolumn{2}{|c|}{ Soccer } & \multicolumn{2}{|c|}{ MGs } & \multicolumn{2}{|c|}{ SSGs } & \multicolumn{2}{|c|}{ FSGs } \\
\hline & Mean & SD & Mean & SD & Mean & SD & Mean & SD & Mean & SD \\
\hline Interest & 5.40 & 0.20 & 5.21 & 0.14 & 5.26 & 1.13 & 5.39 & 1.12 & 5.16 & 1.28 \\
\hline Competence & 5.01 & 0.25 & 4.74 & 0.17 & 4.83 & 1.18 & 4.97 & 1.31 & 4.69 & 1.48 \\
\hline Effort & 4.61 & 0.22 & 4.90 & 0.15 & 4.50 & 1.17 & $4.91 *$ & 1.28 & 4.81 & 1.31 \\
\hline
\end{tabular}

$* p<0.05$ 
Table 2. Summary of Hierarchical Regression Analysis for Variables Predicting MVPA in MGs (N=67)

\begin{tabular}{|c|c|c|c|c|c|c|}
\hline \multirow[t]{2}{*}{ Variable } & \multicolumn{3}{|c|}{ Model 1} & \multicolumn{3}{|c|}{ Model 2} \\
\hline & $\boldsymbol{B}$ & SE B & $\beta$ & $\boldsymbol{B}$ & SE B & $\beta$ \\
\hline Constant & 32.93 & 3.58 & & 38.11 & 4.24 & \\
\hline Sports & 17.63 & 4.32 & $0.45 * *$ & 10.06 & 5.49 & 0.26 \\
\hline Interest/Enjoyment & & & & -5.76 & 2.45 & $-0.36^{*}$ \\
\hline Competence & & & & 2.49 & 1.84 & 0.16 \\
\hline Effort & & & & 4.21 & 2.62 & 0.27 \\
\hline $\mathrm{R}^{2}$ & & 0.20 & & & 0.29 & \\
\hline$F$ for change in $R^{2}$ & & $16.66^{* *}$ & & & 2.36 & \\
\hline
\end{tabular}

Sports was represented as one dummy variable with badminton serving as the reference group; Interest, competence, and effort were centered at their means; $* p<0.05 . * * p<0.01$

Table 3. Summary of Hierarchical Regression Analysis for Variables Predicting MVPA in SSGs (N=67)

\begin{tabular}{|c|c|c|c|c|c|c|}
\hline \multirow[t]{2}{*}{ Variable } & \multicolumn{3}{|c|}{ Model 1} & \multicolumn{3}{|c|}{ Model 2} \\
\hline & B & SE B & $\beta$ & $B$ & SE B & $\boldsymbol{\beta}$ \\
\hline Constant & 35.05 & 3.37 & & 32.36 & 3.00 & \\
\hline Sports & 12.17 & 4.06 & $0.35 * *$ & 16.12 & 3.66 & $0.46^{* *}$ \\
\hline Interest/Enjoyment & & & & -1.05 & 2.06 & -0.07 \\
\hline Competence & & & & 6.56 & 1.44 & $0.53 *$ \\
\hline Effort & & & & -0.35 & 1.86 & -0.27 \\
\hline $\mathrm{R}^{2}$ & & 0.12 & & & 0.36 & \\
\hline$F$ for change in $R^{2}$ & & $8.98 * *$ & & & $7.53 * *$ & \\
\hline
\end{tabular}

Sports was represented as one dummy variable with badminton serving as the reference group; Interest, competence, and effort were centered at their means; ${ }^{*} p<0.05 . * * p<0.01$

Table 4. Summary of Hierarchical Regression Analysis for Variables Predicting MVPA in FSGs (N=67)

\begin{tabular}{|c|c|c|c|c|c|c|}
\hline \multirow[t]{2}{*}{ Variable } & \multicolumn{3}{|c|}{ Model 1} & \multicolumn{3}{|c|}{ Model 2} \\
\hline & $\boldsymbol{B}$ & SE B & $\beta$ & $\boldsymbol{B}$ & SE B & $\boldsymbol{\beta}$ \\
\hline Constant & 33.70 & 3.15 & & 32.82 & 3.08 & \\
\hline Sports & 23.35 & 3.81 & $0.61 * *$ & 24.65 & 3.73 & $0.64 * *$ \\
\hline Interest/Enjoyment & & & & -1.29 & 1.88 & -0.09 \\
\hline Competence & & & & 3.28 & 1.47 & $0.27 *$ \\
\hline Effort & & & & 0.93 & 1.77 & -0.07 \\
\hline $\mathrm{R}^{2}$ & & 0.37 & & & 0.44 & \\
\hline $\mathrm{F}$ for change in $\mathrm{R}^{2}$ & & $37.6^{*}$ & & & 2.48 & \\
\hline
\end{tabular}

Sports was represented as one dummy variable with badminton serving as the reference group; Interest, competence, and effort were centered at their means; $* p<0.05 . * * p<0.01$

tistically significant predictor variables $(p<.01)$. Based on the model, students in soccer classes had 16.12 units MVPA more than those in badminton classes. While holding all the other variables constant, every unit increase in the competence would increase MVPA by 6.56 units. For FSGs (Table 4), the patterns of the regression models were similar with those in SSGs. Sports was a statistically significant variable $(p<.01)$ and it accounted for $37 \%$ of the variance of the dependent variable-MVPA. Students in soccer classes had 23.35 units MVPA more than those in badminton classes did. The whole model accounted for $44 \%$ of the variance of the dependent variable. However, the addition of the variables did not make the change statistically significant. Both the sports $(p<.01)$ and perceived competence variables $(p<.05)$ were statistically significant predictor variables. Based on the model, students in soccer classes had 24.65 units MVPA more than those in badminton classes. While holding all the other variables constant, every unit increase in perceived competence will increase MVPA by 3.28 units.

\section{DISCUSSION}

The discussion will be in accordance with the sequence of the three research questions. These were related to: MVPA 
differences, motivation differences, and using motivation qualities to predict students MVPA in the three different game types within each of the two sports. Students in badminton classes had statistically significantly less MVPA than those in soccer classes. The result aligns with previous studies (e.g., Ainsworth et al., 2011). The previous studies were conducted in regular games abiding by the official rules. Whereas, participants in the current study played the MGs, SSGs, and FSGs in each of the sports. Data in the current study showed that the different game types did not change the overall MVPA pattern in badminton and soccer that players have more MVPA in soccer than they do in badminton. With similar motivation qualities (Table 1), the MVPA difference between the two sports was due to the different nature of the sports. Students at the beginning badminton level have difficulty maintaining longer rallies and they need to retrieve shuttlecocks when the shuttlecocks land. Moreover, students play the game at a relatively slow pace, and they have to wait for the return of shuttlecocks to continue to play. The time associated with frequently retrieving and waiting for the return of shuttlecocks restricted students in the current study from achieving higher MVPA. However, students at the beginning soccer level can keep moving most of the time either for guiding opponents defensively or for moving off the ball offensively. While the soccer players do need to retrieve the balls when they go out of play, this occurred at a much lower frequency because soccer game play is more continuous.

The MVPA results demonstrated that the college students in badminton classes fell short of having more than $50 \%$ MVPA during gameplay even though the counterparts in soccer classes barely achieved the goal (see Figure 1). The findings were not commensurate with previous studies (e.g., Arnett, 2001; Van Acker et al., 2010) where students' MVPA was greater than this recommendation (about 60\% MVPA). Moreover, the participants in the two previous studies accumulated those high MVPA levels from the whole class time. However, the college students in this current study only had their MVPA measured when they played each of the three game types for a continuous 15-minute period. The MVPA that the college students had in the current study was much lower than the MVPA collected during gameplay in other studies (e.g., McCormick et al., 2012; Slingerland et al., 2014). For example, Slingerland et al. (2014) found that boys achieved 74\% MVPA and girls achieved 64\% MVPA during twenty-five-minute small-sided basketball games.

Compared with MVPA findings in other studies, the relatively lower MVPA in the current study may be a result of several differences. First, Van Acker et al. (2010) and Slingerland et al. (2014) collected MVPA data by heart rate monitors, whereas the data for the current study were collected by accelerometers, which have been suggested to be more accurate devices for measuring MVPA (e.g., Heyward \& Gibson, 2014). Second, the participants of the two previous studies were middle school students, whereas the participants in the current study were college students. That said, it is not clear whether these age differences lead to the MVPA differences. After all, adult learners are less active than younger learners (e.g., Douglas et al., 1997; Grunbaum et al., 2002).
The MVPA attained in the three badminton classes was not significantly different. This suggests that PE and sport practitioners can utilize a mixture of MGs, SSGs and/or FSGs when teaching badminton without compromising MVPA. However, it is necessary to point out that badminton students demonstrated the statistically significant better quality of perceived competence and effort/importance in SSGs than they did in MGs and FSGs. When combining both MVPA and motivation data, SSGs were the most optimal game types for badminton classes.

Contrary to the findings by Mallo and Navarro (2008) that students would get significant PA levels when no goalkeepers were present, the university students in the soccer classes had similar MVPA in MGs where no goalkeepers were used as they had in SSGs where goalkeepers were employed. Additionally, and contrary to previous findings in invasion games with middle school students (e.g. Roberts \& Fairclough, 2011), the university students in the soccer classes had the highest MVPA in FSGs. However, they also reported the lowest motivation quality in FSGs. In terms of motivation data, students from badminton classes and soccer classes shared a similar quality of interest/enjoyment, perceived competence, and effort and importance when the data were aggregated across the three game types. It is interesting to find that students demonstrated similar interaction patterns between game types and sports in the perceived competence and effort/importance subscales. In other words, perceived competence and effort/importance are highly associated. For example, students from badminton felt the most competent in SSGs and they made the greatest effort in SSGs. They felt the least competent in MGs and they made the least effort in MGs.

Even though students played the MGs and SSGs on the same sized court, the rules of the MGs prevented students from using smash shots, whereas, in SSGs, students were permitted to use all techniques and tactics. In addition, by the time students played the MGs, the GTA leading the class had not taught the smash shot, thus, it was reasonable to design the MGs without smashes. To have students understand the MGs and to have them more involved, PE and sport practitioners need to emphasize why the specific MGs are necessary and beneficial for the development of techniques and tactics when they first introduce MGs (e.g., Alfieri, Brooks, Aldrich, \& Tenenbaum, 2011). With the stipulations of the importance of MGs, students are more likely to see the objective of the MGs. A good example of using MGs to emphasize the usage of clears and drop shots in badminton is when $\mathrm{PE}$ and sport practitioners can explicitly state the focus of the MGs is for the students to use clears to move their opponents to the deep corners of the court and, subsequently, follow this shot with a drop shot to the front of the court near the net. The purpose of the badminton MGs is to, therefore, find the correct moments to employ drop shots after the clear. Students in the badminton classes reported less quality of perceived competence and effort/importance in FSGs. Based on the first author's previous BIP teaching experience, most students in badminton classes were beginners and this was probably the same for those students in the badminton classes used in this current study. Even though they felt the most comfortable in SSGs, it was likely they found it much harder 
to utilize the techniques and tactics in FSGs with an almost doubled-size playing area. It was also likely that in FSGs students found it harder to place the shuttlecocks accurately on areas close to the sidelines without overhitting the shuttlecocks and to defend their spaces without missing the oncoming shuttlecocks.

Students from soccer classes reported the highest score in perceived competence and effort/importance in MGs where two goals were placed, and no goalkeepers existed. Even though no studies were found on the impact of one extra goal on students' playing behaviors, students were expected to be more involved with the presence of two goals. It was likely that in using two goals they found they could play more offensively and, consequently, found it easier to score. When students scored more points, they felt that they were competent, and they would, as a result, try harder to score. This was also a potential reason why perceived competence data aligned well with effort/importance.

Data showed that students in the soccer classes felt the least competent and perceived they put in the least effort in FSGs even though they had the most MVPA in FSGs. Werner, Bunker, and Thorpe (1996) emphasized that games should be taught sequentially from the least tactical complexity (target games) to the most tactical complexity (invasion games). As one of the most popular invasion games, soccer is one of the most complicated sports in the world (Almond, 1986). Players need to keep running to track the ball and other players to set up offensive opportunities or defensive strategies. Players with the ball also need to keep dribbling it at high speed or use skilled footwork to avoid being tackled by defensive players. This was probably the case for the students in the soccer classes when they played the game on a 120 yards $\mathrm{x} 73$ yards pitch size, which is on the slightly bigger side for a recommended full-size soccer pitch. The game was set at this size because there were markers signifying the size of the full-size pitch on the specific field that was used. Consequently, it was interesting that students in the soccer classes reported the lowest scores in the perceived competence and effort/import scales, but they had the most MVPA in FSGs. Due to the size of the FSGs field, students had longer distance sprints on the field to chase opponents defensively and chase the ball offensively. This was the reason they accumulated more MVPA. At the same time, the field was so large that they found it was much harder to use skills competently and why they presented lower quality of motivation data.

As for the multiple regression analyses, it is not surprising to find that sports is a statistically significant predictor of MVPA in all the models because students in soccer classes had statistically significant more MVPA than those in badminton classes. After adding the motivation data to the model, the full model did explain more variance in MVPA. However, the change of explained variance of the dependent variable was only statistically significant in the SSGs. In the SSGs and FSGs, perceived competence was a statistically significant predictor of MVPA. In other words, students who perceived themselves as more competent in both the SSGs and FSGs were likely to possess greater MVPA in those two game types.
From a motivation perspective, our current results suggest that having students feel competent is the key to enhancing their MVPA. Given the characteristics of students such as current levels of technique and tactical development, gender, social compatibility, and ethnicity, PE and sport practitioners should make continuous modifications to accommodate those characteristics. When students make progress, the games should be harder. When students struggle, the games should be easier. Constant adjustment is the key. For example, Van Acker et al. (2010) utilized the same-gender defense rules in their korfball units, and they believed this rule contributed to the girls possessing higher MVPA levels. The same-gender defense may also work for other games like basketball, soccer, and hockey. In addition, playing badminton against someone of more or less equal skill-level may also result in higher MVPA as rallies will last longer.

To our knowledge, no studies have investigated college students' MVPA and motivation for different sports and different game types systematically. The current study helps PE and sport practitioners develop an increased understanding of the potential influences of sport and game types on students' MVPA and their motivations. Thus, PE and sport practitioners can accordingly adjust the use of the three game types to balance students' MVPA and motivations. Conflicting with previous findings that students had low MVPA when FSGs were utilized the most, students in soccer classes had the most MVPA in FSGs. Researchers found that adult learners were less active than younger learners (e.g., Douglas et al., 1997; Grunbaum et al., 2002). With the participants in the previous studies middle-school students (e.g., Roberts \& Fairclough, 2011) and the participants in the current studies adult learners, it is not clear whether the MVPA difference is caused by the age disparity.

The three IMI motivation subscales were utilized to predict students' MVPA. It was surprising to find that interest/ importance was a statistically significant negative predictor of MVPA in MGs and a negative predictor in SSGs and FSGs. Since the overall reported scores for the interest/enjoyment were over 5.2 out of 7, the assumption for this situation is that students who had less MVPA also viewed the games were interesting. Further studies are needed to verify this claim. Effort/ importance was not a statistically significant predictor in all the three-game types. However, perceived competence was a statistically significant predictor of MVPA in both SSGs and FSGs. A student with higher perceived competence was predicted to gain greater MVPA. Moreover, a student who reported higher perceived competence was likely to put in greater effort when playing the games. Even though students in soccer classes attained the most MVPA in FSGs, it does not mean that $\mathrm{PE}$ and sport practitioners should mainly utilize FSGs as the main learning organizer for soccer, after all, the students also demonstrated the lowest motivation qualities across all subscales in FSGs. The principle of exaggeration (i.e., Bunker \& Thorpe, 1986) rationalized the utilization of MGs, which stated that changing game structures, such as rules, equipment, and play space, to promote and exaggerate a particular aspect of a game. The principle of representation (i.e., Bunker \& Thorpe, 1986) provided the theoretical support for using SSGs, which 
stated that SSGs structured to suit the age and/or experience of the players. The SSGs are developed to contain the same tactical structures of the adult game but are played with adaptations to suit players' characteristics. We must realize that PE classes are not only about MVPA, they are also about motivation. Even though students in the soccer classes had the most MVPA in FSGs, they also reported the lowest motivation qualities at the perceived competence and effort/importance. Thus, we should refute the idea that $\mathrm{PE}$ and sport practitioners should mainly use FSGs as the main learning organizer. It is clearer to refute the idea when it comes to badminton classes, given the fact that students in badminton had the best MVPA (even though not statistically significant) and the best motivation qualities in the perceived competence and effort/importance subscales in SSGs instead of FSGs. Even though students in soccer may not have the most MVPA in MGs and SSGs, they could perform more technical requirements in MGs and SSGs when the pitches were reduced (Bell et al., 2013; Platt et al., 2001).

The current study had several strengths. First, accelerometers were utilized to objectively measure MVPA. Second, the quasi-experimental design enables the researchers to conduct the study in a non-interference and natural teaching and learning environment. Finally, the researchers systematically investigated the MVPA and motivation not only in different game types but also in two sports from different game categories.

There were several limitations in the study. First, while the current study adds to the literature on BIP/HEPAP studies, further research with adult learners is needed to further confirm the results of the study. Second, the badminton and soccer classes were general elective classes, and there were no levels of skill differentiation in any of the classes studied. Therefore, it may have been possible there were more intermediate players in one class than in another class. Gender effects were not examined even though the ratio of genders were almost equal since six of the 23 participants in the badminton classes were females and eight of 48 participants in the soccer classes were female. Third, no student learning data were included in this study. Future studies could include authentic assessment data to demonstrate that students learned in addition to gaining MVPA and increasing the quality of their motivation. Finally, due to the quasi-experimental design, we did not manipulate the game sequence, which was fixed in the order of MGs-SSGs-FSGs.

\section{CONCLUSION}

The key findings of the study were that PE and sport practitioners should find the most parsimonious balance between MVPA and motivation through adjusting games to ensure they meet the developmental needs to their students. One way this can be done according to the results of this current study is to manipulate the field/court size and dimensions for MGs and SSGs. Additional ways could be to consider player numbers (i.e., 1 vs. 1,2 vs. 1,2 vs. 2,3 vs. 2,3 vs. 3 , and so on) and specific game rules (i.e., players assigned to specific zones, using different goals, using target zones for winning shots in badminton, etc.).

\section{REFERENCES}

Ainsworth, B. E., Haskell, W. L., Herrmann, S. D., Meckes, N., Bassett Jr, D. R., Tudor-Locke, C., \& Leon, A. S.(2011). 2011 Compendium of physical activities: a second update of codes and MET values. Medicine and Science in Sports and Exercise, 43(8), 1575-1581.

Alfieri, L., Brooks, P. J., Aldrich, N. J., \& Tenenbaum, H. R. (2011). Does discovery-based instruction enhance learning?.American Psychological Association, 103(1), 1-18

Almond, L. (1986). Reflecting on themes: A games classification. Rethinking games teaching. (D. Bunker, R. Thorpe, \& L. Almond, Eds.). England: University of Technology, Loughborough, Department of Physical Education and Sports Science.

American College Health Association. (2012, June). Healthy Campus 2020. Retrieved from http://www.acha.org/ HealthyCampus/index.cfm

Arnett, M. G. (2001). The effect of sport-based physical education lessons on physical activity. Physical Educator, 58(3), 158-167.

Bell, K., Johnson, T. G., Shimon, J., \& Bale, J. (2013). The effects of game size on the physical activity levels and ball touches of elementary school children in physical education. Journal of Kinesiology and Wellness. 1, 1-5

Bunker, D., \& Thorpe, R. (1982). A model for the teaching of games in secondary schools. Bulletin of Physical Education, 18(1), 5-8.

Bunker, D., \& Thorpe, R. (1986). The curriculum mode. Rethinking games teaching. (R. Thorpe, D. Bunker, \& L. Almond, Eds.). Loughborough, UK: University of Technology, Department of Physical Education and Sports Science.

Carroll, B., \& Loumidis, J. (2001). Children's perceived competence and enjoyment in physical education and physical activity outside of school. European Physical Education Review, 7(1), 24-43.

Coutts AJ. Murphy AJ. Dascombe BJ. (2004). The effect of direct supervision of a strength coach on measures of muscular strength and power in young rugby league players. The Journal of Strength and Conditioning Research, 18(2), 157-164.

Crouter, S. E., Schneider, P. L., Karabulut, M., \& Bassett Jr, D. R. (2003). Validity of ten electronic pedometers for measuring steps, distance, and kcals. Medicine and Science in Sports and Exercise, 35(5), 283-288

Deci, E. L., Eghrari, H., Patrick, B. C., \& Leone, D. R. (1994). Facilitating internalization: The self-determination theory perspective. Journal of Personality, 62(1), 119-142

Deci, E. L., \& Ryan, R. M. (1985). The general causality orientations scale: Self-determination in personality. Journal of Research in Personality, 19(2), 109-134.

Deci, E. L., \& Ryan, R. M. (2003). Intrinsic motivation inventory. Self-determination theory. 267

Douglas, K. A., Collins, J. L., Warren, C., Kann, L., Gold, R., Clayton, S., \& Kolbe, L. J. (1997). Results from the 1995 national college health risk behavior survey. Journal of American College Health, 46(2), 55-67.

Eston, R. G., Rowlands, A. V., \& Ingledew, D. K. (1998). Validity of heart rate, pedometry, and accelerometry for 
predicting the energy cost of children's activities. Journal of Applied Physiology, 84(1), 362-371.

Erdfelder, E., Faul, F., \& Buchner, A. (1996). GPOWER: A general power analysis program. Behavior Research Methods, Instruments, and Computers, 28(1), 1-11.

Gabbett, T. J. (2002). Training injuries in rugby league: an evaluation of skill-based conditioning games. The Journal of Strength and Conditioning Research, 16(2), 236-241.

Grunbaum, J. A., Kann, L., Kinchen, S. A., Williams, B., Ross, J. G., Lowry, R., \& Kolbe, L. (2002). Youth risk behavior surveillance-United States, 2001. Journal of School Health, 72(8), 313-328.

Hannon, J. C., \& Ratliffe, T. (2005). Physical activity levels in coeducational and single-gender high school physical education settings. Journal of Teaching in Physical Education, 24(2), 149-164.

Harvey, S., \& Jarrett, K. (2014). A review of the game-centered approaches to teaching and coaching literature since 2006. Physical Education and Sport Pedagogy, 19(3), 278-300.

Harvey, S., Smith, M. L., Song, Y., Robertson, D., Brown, R., \& Smith, L. R. (2016). Gender and School-Level Differences in Students' Moderate and Vigorous Physical Activity Levels When Taught Basketball Through the Tactical Games Model. Journal of Teaching in Physical Education, 35(4), 349-357.

Harvey, S., Song, Y., Baek, J. H., \& van der Mars, H. (2015). Two sides of the same coin: Student physical activity levels during a game-centered soccer unit. European Physical Education Review, 22(4), 411-429

Healthy People 2020. Retrieved from https://www.healthypeople.gov/2020/topics-objectives/topic/physical-activity

Heyward, V. H., \& Gibson, A. (2014). Advanced fitness assessment and exercise prescription $7^{\text {th }}$ edition. In A.N.

Tocco; K.Matz; \& S.Huls (Eds), Using technology to promote physical activity (pp.63-68).Champaign, IL: Human kinetics.

Institute of Medicine. (2013). Educating the student body: Taking physical activity and physical education to school. In H.W.Kohl III \& H.D. Cook (Eds.), Status and trends of physical activity behaviors and related school policies (pp.32-65). Washington, D.C. The National Academies Press

Mallo, J., \& Navarro, E. (2008). Physical load imposed on soccer players during small-sided training games. Journal of Sports Medicine and Physical Fitness, 48(2), 166171.

McAuley, E., Duncan, T., \& Tammen, V. V. (1989). Psychometric properties of the Intrinsic Motivation Inventoryin a competitive sport setting: A confirmatory factor analysis. Research Quarterly for Exercise and Sport, 60(1), 48-58.

Meeteer, W., Housner, L., Bulger, S., Hawkins, A., \& Wiegand, R. (2012). Applying Sport Education in university basic instruction courses. In P. Hastie (Eds.), Sport Edu- cation international perspectives (pp.58-72). NewYork, NY: Routledge

Miller, A., Christensen, E. M., Eather, N., Sproule, J., Annis-Brown, L., \& Lubans, D. R. (2015).The PLUNGE randomized controlled trial: Evaluation of a gamesbased physical activity professional learning program in primary school physical education. Preventive Medicine, 74, 1-8.

Moore, L. L., Lombardi, D. A., White, M. J., Campbell, J. L., Oliveria, S. A., \& Ellison,R. C. (1991). Influence of parents' physical activity levels on activity levels of young children. The Journal of pediatrics, 118(2), 215-219.

Platt, D., Maxwell, A., Horn, R., Williams, M., \& Reilly, T. (2001). Physiological and technical analysis of 3 vs. 3 and 5 vs. 5 youth football matches. Insight, 4(4), 23-24.

Rampinini E. Impellizzeri FM. Castagna C. Abt G. Chamari K. Sassi A. Marcora SM. (2007). Factors influencing physiological responses to small-sided soccer games. Journal of Sports Sciences, 25(6), 659-666.

Reilly, T., \& White, C. (2005). Small-sided games as an alternative to interval training for soccer players. Science and football $V, 355-358$

Ryan, R. M. (1982). Control and information in the intrapersonal sphere: An extension of cognitive evaluation theory. Journal of Personality and Social Psycholo$g y, 43(3), 450-462$.

Roberts, S., \&Fairclough, S. (2011). Observational analysis of student activity modes, lesson contexts and teacherinteractions during games classes in high school (1116 years) physical education. European Physical Education Review, 17(2), 255-268.

Sparling, P. B., \& Snow, T. K. (2002). Physical activity patterns in recent college alumni.Research Quarterly for Exercise \& Sport, 73, 200-205.doi:10.1080/02701367. 2002.10609009

Stapleton, D. T., Taliaferro, A. R., \& Bulger, S. M. (2017). Teaching an old dog new tricks: Past, present, and future priorities for higher education physical activity programs. Quest, 1-18.

Sun, H., \& Chen, A. (2010). A pedagogical understanding of the self-determination theory in physical education. Quest, 62(4), 364-384.

Van Acker, R., Carreiro da Costa, F., De Bourdeaudhuij, I., Cardon, G., \& Haerens, L. (2010). Sex equity and physical activity levels in coeducational physical education: exploring the potential of modified game forms. Physical Education and Sport Pedagogy, 15(2), 159-173.

Werner, P., Thorpe, R., \& Bunker, D. (1996). Teaching games for understanding: Evolution of a model. Journal of Physical Education, Recreation and Dance, 67(1), 28-33.

Yelling, M., Penney, D., \& Swaine, I. L. (2000). Physical activity in physical education: A case study investigation. European Journal of Physical Education, 5(1), 45-66. 